\title{
The efficacy of probiotics in patients with severe COVID-19
}

\author{
Huaqi Wang ${ }^{1}$, Yunfei Wang ${ }^{1}$, Chunya Lu ${ }^{1}$, Lingxiao Qiu ${ }^{1}$, Xiangjin Song ${ }^{1}$, Hongxia Jia ${ }^{1}$, Dong Cui ${ }^{2}$, \\ Guojun Zhang ${ }^{1}$
}

${ }^{1}$ Department of Respiratory and Critical Care Medicine, The First Affiliated Hospital of Zhengzhou University, Zhengzhou, China; ${ }^{2}$ Department of Respiratory and Critical Care Medicine, Xinyang Fifth People's Hospital, Xinyang, China

Contributions: (I) Conception and design: H Wang, Y Wang; (II) Administrative support: None; (III) Provision of study materials or patients: L Qiu, G Zhang; (IV) Collection and assembly of data: C Lu, X Song; (V) Data analysis and interpretation: H Jia, G Zhang; (VI) Manuscript writing: All authors; (VII) Final approval of manuscript: All authors.

Correspondence to: Huaqi Wang. Department of Respiratory and Critical Care Medicine, The First Affiliated Hospital of Zhengzhou University, Zhengzhou, China. Email: wanghuaqi2004@126.com; Guojun Zhang. Respiratory Department, The First Affiliated Hospital of Zhengzhou University, No.1 Jianshe Road, Zhengzhou 450052, China. Email: gjzhangzzu@126.com.

\begin{abstract}
Background: To examine the incidence of diarrhea in severe and critical coronavirus disease 2019 (COVID-19) patients, and to observe the efficacy and prognosis of probiotic use in such patients.

Methods: A retrospective study was conducted to investigate the symptoms and incidence of diarrhea in 156 cases of COVID-19 confirmed by the First Affiliated Hospital of Zhengzhou University and the Xinyang Fifth People's Hospital, China. A total of 58 cases of severe and critical COVID-19 were identified and divided into the treatment group or the control group. The control group was given standard treatment according to the Protocols for Diagnosis and Treatment of COVID-19: Prevention, Control, Diagnosis and Management. Patients in the treatment group were administered oral probiotics as well as the standard treatment. The 2 groups were compared in terms of nutritional status (serum albumin), improvement of diarrhea symptoms, changes in inflammatory condition [procalcitonin (PCT) and C-reactive protein (CRP)], the time taken to register a negative result for respiratory tract pathogens on the nucleic acid test, and changes to white blood cell and lymphocyte cell counts.
\end{abstract}

Results: In this study cohort, diarrhea was detected in 15.38\% (24/156) of COVID-19 patients. The incidence of diarrhea in patients with mild and moderate COVID-19 was approximately 8.16\% (8/98), and the incidence of diarrhea in severe and critically ill patients was approximately $27.59 \%$ (16/58). In patients with severe and critical COVID-19, probiotic treatment obviously shortened the duration of diarrhea. Furthermore, compared with the control group, patients treated with probiotics showed a significantly reduced time to achieving a negative nucleic acid test and the inflammation indexes including PCT and CRP were significantly reduced $(\mathrm{P}<0.05)$.

Conclusions: The incidence of diarrhea in severe and critically ill COVID-19 patients was significantly higher than that in patients with mild and moderate COVID-19. Probiotics may have a good supporting role in the treatment of patients with COVID-19 and its early application is recommended.

Keywords! Probiotics; coronavirus disease 2019 (COVID-19); diarrhea

Submitted Oct 08, 2021. Accepted for publication Dec 17, 2021.

doi: 10.21037/apm-21-3373

View this article at: https://dx.doi.org/10.21037/apm-21-3373

\section{Introduction}

In December 2019, a number of viral pneumonia cases were identified in Wuhan, in the Hubei province of China. The National Health Commission of China named this novel viral infection the "new coronavirus pneumonia" (1), and this is now known as coronavirus disease 2019 (COVID-19) (2). By early March 2020, COVID-19 had spread rapidly worldwide and the World Health Organization (WHO) Director, General Tedros Adhanom Ghebreyesus, 
declared the COVID-19 epidemic had "global pandemic characteristics" (3). China launched a concerted control, treatment, and prevention effort with much success.

Interestingly, analysis of the clinical characteristics of COVID-19 revealed that diarrhea occurred in approximately $3.8 \%$ of cases (4). In fact, diarrhea was often reported as the first symptom of COVID-19 (5). Novel coronavirus entry into host cells is mediated by the interaction between envelope-anchored viral spike proteins and host receptors [composed of angiotensin converting enzyme 2 (ACE2)]. Infectivity of the virus depends primarily on the affinity for binding to the ACE2 receptor. Studies have shown that ACE2 is highly expressed in the absorbing cells of the upper esophagus, ileum and colon, which increases the chance of virus invasion. In addition, viral infection may cause changes in intestinal permeability, resulting in malabsorption of intestinal cells (6). A large number of spectral antibiotics will aggravate the symptoms of diarrhea. Intestinal flora can affect a variety of respiratory diseases, including asthma, acute and chronic respiratory infections, through the enteric-lung axis (7). Studies have shown that the composition and diversity of intestinal microorganisms in patients with viral pneumonia have also changed. Wang et al. (8) observed a decrease in the number of intestinal lactobacillus in a mouse model infected with influenza virus. Groves et al. (9) used the mouse model of respiratory syncytial virus and influenza virus, and observed an increase in the number of intestinal bacteroidetes and a decrease in the abundance of Firmicutes phyla in mice. The latest novel Coronavirus study shown that novel coronavirus also affects intestinal flora, with significantly lower bacterial diversity and significantly increased relative abundance of opportunistic pathogens in COVID-19 patients compared to healthy controls (10). Drugs that regulate gut flora play an important role in the treatment of patients with COVID-19. Therefore, this current study investigated the incidence of diarrhea in COVID-19 patients and determined the therapeutic value of administering probiotics to COVID-19 patients. A total of 156 patients with COVID-19 admitted to the First Affiliated Hospital of Zhengzhou University in Henan or the Xinyang Fifth People's Hospital were enrolled in this study. There were 58 cases of severe and critical COVID-19. This study was conducted to compare the therapeutic efficacy of probiotic therapy and the Protocols for Diagnosis and Treatment of COVID-19: Prevention, Control, Diagnosis and Management. The incidence of diarrhea, as well as the inflammatory markers lever were analyzed before and after probiotic treatment and the therapeutic value of probiotic treatment for severe COVID-19 patients was evaluated. We present the following article in accordance with the STROBE reporting checklist (available at https://dx.doi. org/10.21037/apm-21-3373).

\section{Methods}

\section{Patients}

A total of 156 patients were enrolled in this study. The diagnostic criteria of COVID-19 were in accordance with the Diagnostic Criteria and Clinical Classification of COVID-19 issued by the Health Committee of China (7th Edition) (11). All procedures performed in this study involving human participants were in accordance with the Declaration of Helsinki (as revised in 2013). The study was approved by the First Affiliated Hospital of Zhengzhou University (No.: 2020-KY-056). Individual consent for this retrospective analysis was waived.

The clinical characteristics of the patients and the incidence of diarrhea were documented. Patients were randomly divided into a control group and a treatment group. Patients in the control group were treated according to the Protocols for Diagnosis and Treatment of COVID-19: Prevention, Control, Diagnosis and Management. Patients in the treatment group were administered oral adjuvant therapy consisting of routine intestinal probiotics in addition to the standard treatment given to the control group. Probiotic administration protocol: Bifidobacterium lactobacillus triplex live tablet (Abbreviation: gold bifidum, Inner Mongolia Shuangqi Pharmaceutical Co. LTD, China. National drug approval: S19980004, $0.5 \mathrm{~g} /$ tablet. Each tablet should contain no less than $0.5 \times 10^{7} \mathrm{CFU}$ of live Bifidobacterium longum; live Lactobacillus bulgaricus and Streptococcus thermophilus should not be lower than $\left.0.5 \times 10^{6} \mathrm{CFU}\right), 4$ pieces at a time, 3 times a day.

\section{Observation indexes}

The nutritional status assessed by serum albumin (ALB) levels; the frequency of diarrhea; the inflammatory status as assessed by the inflammatory markers procalcitonin (PCT) and C-reactive protein (CRP); the time required to achieve a negative result on nucleic acid tests; and the leukocyte, lymphocyte, and neutrophil counts were examined in all patients prior to intervention and 1 week after intervention. 
Table 1 The clinical characteristics of the COVID-19 patients in this study

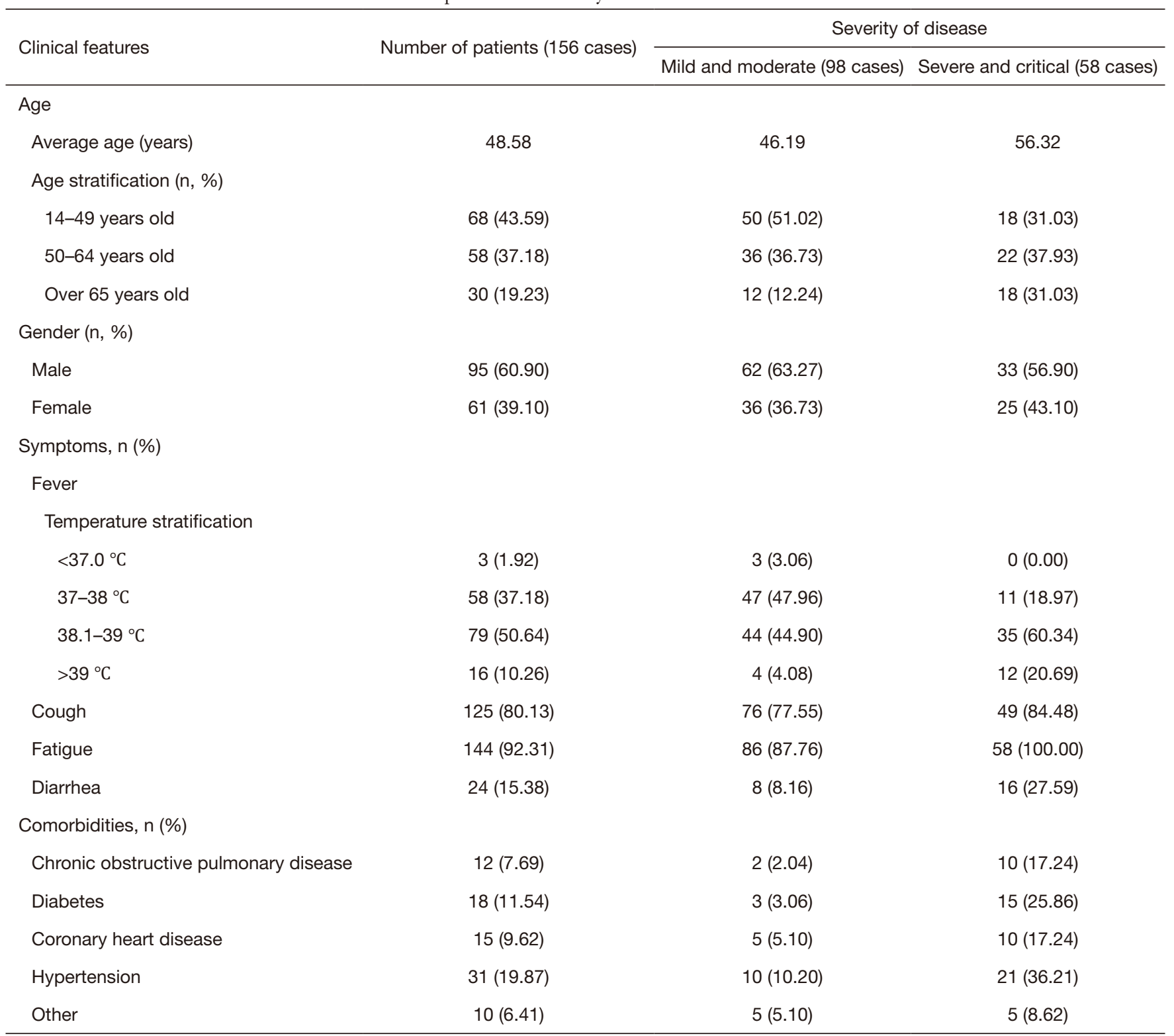

COVID-19, coronavirus disease 2019.

\section{Statistical analysis}

The SPSS 25 software was used for all statistical analyses. The $t$-test was applied for normally distributed quantitative data and the $\chi^{2}$ test was applied to qualitative data. The nonparametric rank sum test was used to assess data that did not conform to normal distribution and the significance level was set to $\alpha=0.05$.

\section{Results}

\section{Patient characteristics}

A total of 156 COVID-19 patients were enrolled in this study, including 98 patients with mild to moderate COVID-19 and 58 cases of severe to critical COVID-19 (Table 1). The total incidence of diarrhea was approximately $15.38 \%$ (24/156). Approximately 8.16\% (8/98) of patients 
Table 2 A comparison of the incidence of diarrhea between patients with severe to critical COVID-19 and patients with mild to moderate disease

\begin{tabular}{lccc}
\hline \multirow{2}{*}{ Group } & \multicolumn{2}{c}{ Diarrhea } & Chi $^{2}$ value \\
\cline { 2 - 4 } & Yes $(\mathrm{n})$ & No $(\mathrm{n})$ & 10.559 \\
\hline Mild to moderate & 8 & 90 & \\
Severe to critical & 16 & 42 & \\
Total $(\mathrm{n})$ & 24 & 132 & \\
\hline
\end{tabular}

Table 3 The efficacy of probiotic treatment in patients with severe to critical COVID-19 ( $n=16)$

\begin{tabular}{lccc}
\hline Group & Average value of the duration of diarrhea & Standard deviation & T value \\
\hline Treatment $(n=10)$ & 8.60 & 2.31 & -2.41 \\
Control $(n=6)$ & 11.50 & 2.35 & 0.030 \\
\hline
\end{tabular}

COVID-19, coronavirus disease 2019.

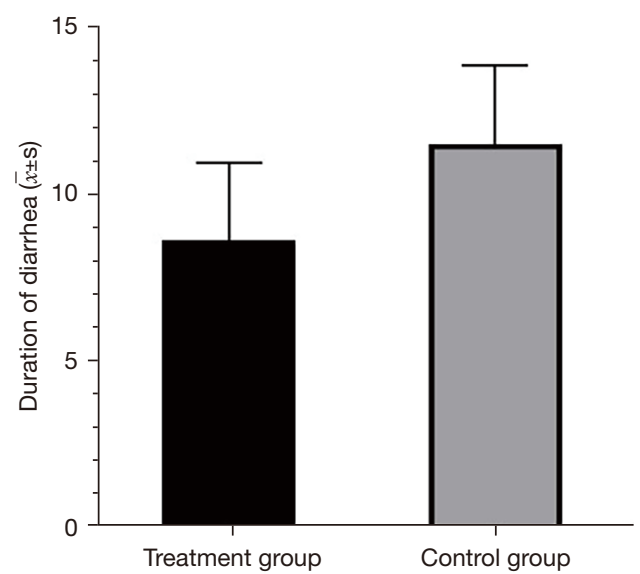

Figure 1 The duration of diarrhea in severe and critically ill patients with COVID-19 who were treated with probiotics. COVID-19, coronavirus disease 2019.

with mild to moderate COVID-19 experienced diarrhea compared to a significantly higher incidence of $27.59 \%$ $(16 / 58)$ in patients with severe to critical disease $(\mathrm{P}<0.05$; Table 2).

The 58 patients with severe and critical COVID-19 were treated with the Protocols for Diagnosis and Treatment of COVID-19: Prevention, Control, Diagnosis and Management (the control group; $\mathrm{n}=35$ ), or the Protocols for Diagnosis and Treatment of COVID-19: Prevention, Control, Diagnosis and Management together with probiotics (the treatment group; $\mathrm{n}=23$ ).

\section{Duration of diarrhea}

Of the 58 patients with severe and critical COVID-19, 16 presented with symptoms of diarrhea. Probiotics was administered to10 patients (the probiotics group) and the remaining 6 patients were only given the Protocols for Diagnosis and Treatment of COVID-19: Prevention, Control, Diagnosis and Management (the control group). The duration of diarrhea in the probiotics group was significantly shorter than that in the control group $(\mathrm{P}<0.05$; Table 3; Figure 1). The patient's diarrhea was effectively controlled.

\section{Laboratory indicators}

Patient laboratory indexes were assessed before therapy and one after week standard COVID-19 treatment protocols and/or probiotic treatment. The levels of the inflammatory markers PCT and CRP were significantly lower in the treatment group compared to the control group $(\mathrm{P}<0.05)$. The plasma albumin levels and lymphocyte counts were significantly higher in patients treated with probiotics compared to patients in the control group $(\mathrm{P}<0.05)$. The time to achieving a negative nucleic acid test result was significantly shorter than that of the control group $(\mathrm{P}<0.05)$. There were no significant differences in the white blood 
Table 4 Laboratory indexes in severe COVID-19 patients treated with or without probiotics (Non-normal distribution data)

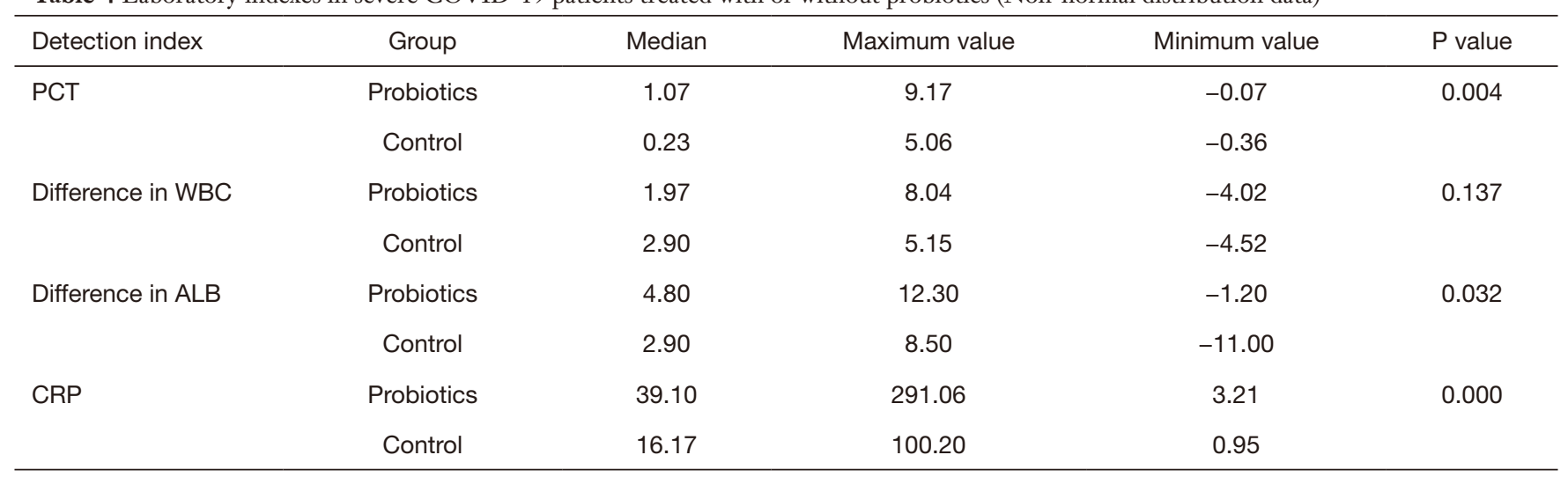

$\mathrm{n}=23$ for the treatment group; $\mathrm{n}=35$ for the control group. COVID-19, coronavirus disease 2019; PCT, procalcitonin; WBC, white blood cell; ALB, albumin; CRP, C-reactive protein.

Table 5 Changes in the laboratory indexes in the treatment group and the control group (normal distribution data)

\begin{tabular}{|c|c|c|c|c|c|}
\hline Detection Index & Group & Average value & Standard deviation & T value & $P$ value \\
\hline Difference in neutrophil count & Control & 10.58 & 8.99 & & \\
\hline \multirow[t]{2}{*}{ Nucleic acid negative time } & Treatment & 12.91 & 0.90 & -2.79 & 0.007 \\
\hline & Control & 16.57 & 0.88 & & \\
\hline Difference in lymphocyte count & Control & 0.55 & 0.08 & & \\
\hline
\end{tabular}

$n=23$ for the treatment group; $n=35$ for the control group.

cell count nor the neutrophil count between the two groups $(\mathrm{P}>0.05 ;$ Tables 4,5).

\section{Discussion}

Normal intestinal microflora consists of thousands of bacteria that naturally colonized the gastrointestinal tract (12) and these play an important role in maintaining good health. Intestinal microflora can regulate the immune response of the body, competitively inhibit the colonization of pathogenic bacteria, produce immune tolerance to symbiotic bacteria, maintain the stability of the intestinal environment, and decompose and synthesize nutrients for absorption and utilization by the body (13). The structural components of gastrointestinal bacteria, as well as their metabolites, can enter the circulatory system and affect the environment outside the intestine. For example, the cell wall components of gram-negative bacteria, as well as lipopolysaccharides (LPS) and bacterial metabolite short chain fatty acids, can exert regulatory effects on the immune system $(9,14)$. Studies have shown that Lactobacillus acidophilus $L-92, L$. rbamnophilus $G G$, and other probiotics can enhance the body's immune and antiviral response $(15,16)$. Probiotics can inhibit the growth of harmful bacteria by maintaining a weak acid environment in the gut, exerting antibacterial, anti-inflammatory, and even anti-tumor effects. Indeed, the current study found that the lymphocyte count in patients treated with probiotics was significantly higher than that in the control group. However, there was no significant difference in the neutrophil count between the two groups, suggesting that the probiotics had some effect on improving the lymphatic function of patients, but had no correlation with the function of neutrophils.

In this study, 156 confirmed cases of COVID-19 were diagnosed and the overall incidence of diarrhea was $15.38 \%$ (24/156). The incidence of diarrhea in severe to criticallyill patients was significantly higher than that in patients 
with mild to moderate disease $(8.16 \%$ vs. $27.59 \%)$. The incidence of diarrhea is higher in patients. It is possible that diarrhea may not only be caused by the actual coronavirus infection, but also by the side effects of antiviral drugs or the use of high doses of hormones and antibiotics during treatment.

Previous studies have demonstrated that the acute exacerbation of COVID-19 patients is more related to the "inflammatory storm" caused by COVID-19 infection (17-19). In our study cohort, administration of probiotics to patients with severe COVID-19 significantly reduced the duration of diarrhea and improved the nutritional status compared to control patients. Furthermore, the time to achieving a negative nucleic acid test result was significantly shorter than that of the control group. Probiotic treatment also reduced the inflammatory indexes PCT and CRP significantly compared to the control group. These results suggested that administration of probiotics to patients with severe COVID-19 can effectively reduce the inflammatory reaction, shorten the time of nucleic acid conversion, and improve the prognosis of patients.

\section{Acknowledgments}

Funding: This study was supported by the first batch of Novel Coronavirus Emergency Prevention and Control Project (No.201100310600).

\section{Footnote}

Reporting Checklist: The authors have completed the STROBE reporting checklist. Available at https://dx.doi. org/10.21037/apm-21-3373

Data Sharing Statement: Available at https://dx.doi. org/10.21037/apm-21-3373

Conflicts of Interest: All authors have completed the ICMJE uniform disclosure form (available at https://dx.doi. org/10.21037/apm-21-3373). The authors have no conflicts of interest to declare.

Ethical Statement: The authors are accountable for all aspects of the work in ensuring that questions related to the accuracy or integrity of any part of the work are appropriately investigated and resolved. All procedures performed in this study involving human participants were in accordance with the Declaration of Helsinki (as revised in 2013). The study was approved by the First Affiliated Hospital of Zhengzhou University (No.: 2020-KY-056). Individual consent for this retrospective analysis was waived.

Open Access Statement: This is an Open Access article distributed in accordance with the Creative Commons Attribution-NonCommercial-NoDerivs 4.0 International License (CC BY-NC-ND 4.0), which permits the noncommercial replication and distribution of the article with the strict proviso that no changes or edits are made and the original work is properly cited (including links to both the formal publication through the relevant DOI and the license). See: https://creativecommons.org/licenses/by-nc-nd/4.0/.

\section{References}

1. Liu YC, Kuo RL, Shih SR. COVID-19: The first documented coronavirus pandemic in history. Biomed J 2020;43:328-33.

2. Harapan H, Itoh N, Yufika A, et al. Coronavirus disease 2019 (COVID-19): A literature review. J Infect Public Health 2020;13:667-73.

3. Shi Y, Wang G, Cai XP, et al. An overview of COVID-19. J Zhejiang Univ Sci B 2020;21:343-60.

4. Jiang F, Deng L, Zhang L, et al. Review of the Clinical Characteristics of Coronavirus Disease 2019 (COVID-19). J Gen Intern Med 2020;35:1545-9.

5. Song $\mathrm{Y}$, Liu $\mathrm{P}$, Shi XL, et al. SARS-CoV-2 induced diarrhoea as onset symptom in patient with COVID-19. Gut 2020;69:1143-4.

6. D'Amico F, Baumgart DC, Danese S, et al. Diarrhea During COVID-19 Infection: Pathogenesis, Epidemiology, Prevention, and Management. Clin Gastroenterol Hepatol 2020;18:1663-72.

7. Marsland BJ, Trompette A, Gollwitzer ES. The Gut-Lung Axis in Respiratory Disease. Ann Am Thorac Soc 2015;12 Suppl 2:S150-6.

8. Wang J, Li F, Wei H, et al. Respiratory influenza virus infection induces intestinal immune injury via microbiotamediated Th17 cell-dependent inflammation. J Exp Med 2014;211:2397-410.

9. Groves HT, Cuthbertson L, James P, et al. Respiratory Disease following Viral Lung Infection Alters the Murine Gut Microbiota. Front Immunol 2018;9:182.

10. Li F, Lu H, Li X, et al. The impact of COVID-19 on intestinal flora: A protocol for systematic review and meta analysis. Medicine (Baltimore) 2020;99:e22273.

11. Epidemiology Working Group for NCIP Emergency 
Response, Chinese Center for Disease Control and

Prevention. Rapid investigation plan of clinical courses and key diagnosis and treatment equipment requirements for the patients of the 2019 Novel Coronavirus Pneumonia (COVID-19). Zhonghua Liu Xing Bing Xue Za Zhi 2020;41:296.

12. D'Argenio V, Salvatore $F$. The role of the gut microbiome in the healthy adult status. Clin Chim Acta 2015;451:97-102.

13. van Nood E, Speelman P, Nieuwdorp M, et al. Fecal microbiota transplantation: facts and controversies. Curr Opin Gastroenterol 2014;30:34-9.

14. Goto H, Sagitani A, Ashida N, et al. Anti-influenza virus effects of both live and non-live Lactobacillus acidophilus L-92 accompanied by the activation of innate immunity. Br J Nutr 2013;110:1810-8.

15. Harata G, He F, Hiruta N, et al. Intranasal administration

Cite this article as: Wang $\mathrm{H}$, Wang $\mathrm{Y}$, Lu C, Qiu L, Song X, Jia H, Cui D, Zhang G. The efficacy of probiotics in patients with severe COVID-19. Ann Palliat Med 2021;10(12):1237412380. doi: 10.21037/apm-21-3373 of Lactobacillus rhamnosus GG protects mice from $\mathrm{H} 1 \mathrm{~N} 1$ influenza virus infection by regulating respiratory immune responses. Lett Appl Microbiol 2010;50:597-602.

16. Mu C, Yang Y, Zhu W. Crosstalk Between The Immune Receptors and Gut Microbiota. Curr Protein Pept Sci 2015;16:622-31.

17. Mehta P, McAuley DF, Brown M, et al. COVID-19: consider cytokine storm syndromes and immunosuppression. Lancet 2020;395:1033-4.

18. Ma J, Xia P, Zhou Y, et al. Potential effect of blood purification therapy in reducing cytokine storm as a late complication of critically ill COVID-19. Clin Immunol 2020;214:108408.

19. Vaninov N. In the eye of the COVID-19 cytokine storm. Nat Rev Immunol 2020;20:277. 